\title{
Taxonomic studies on Cheloninae (Hymenoptera, Braconidae) fauna of Northeastern Anatolia region (Ardahan, Erzurum, Iğdır and Kars) of Turkey
}

\author{
Ahmet BeyarsLan \\ Department of Biology, Faculty of Science, Trakya University, Edrine, Turkey. \\ e-mail: abeyars@gmail.com
}

\begin{abstract}
Beyarslan, A.: Taxonomic Studies on Cheloninae (Hymenoptera, Braconidae) Fauna of Northeastern Anatolia Region (Ardahan, Erzurum, I $\breve{g} d ı r$ and Kars) of Turkey.

Abstract: In order to determine Cheloninae (Hymenoptera, Braconidae) fauna of Turkey, adult specimens were collected from different habitats of Ardahan, Erzurum, Iğdır and Kars provices using sweeping nets, Malaise and light traps. Obtained material was prepared and according modern taxonomic rules and appropriately labeled. Relevant literature and comparison material available in our collection were used for taxonomical examination of the material. The properties of the localities and collection dates were also given. A total of 32 species from 3 genera could be recorded. Nine of these species are the first record for the fauna of Turkey.
\end{abstract}

Keywords: koinobiont, faunistic, parasitoid, Chelonini, Turkey.

\section{Introduction}

Cheloninae Foerster, 1863 (Hymenoptera: Braconidae) is represented worldwide with 1523 species described and include the tribes Chelonini Foerster, 1863, Adeliini Viereck, 1918, Odontosphaeropygini Zettel, 1990 and Phanerotomini Baker, 1926. The largest tibe of the subfamily is Chelonini and includes 1077 species (YU et al. 2016). This subfamily is characterized by the following apomorphic characters: Metasomal carapace, short and wide radial cell, the presence of the postpectal carina, eyes with setae, which are often intensely seen (van ACHTERBERG 1976). Final instar larvae with thin and cylindrical mandibles and usually the presence of indeterminate hypostomal sutures (KAESLIN et al. 2005). Members of the Cheloninae are egg-larval solitary endoparasitoids and koinobionts of Lepidoptera especially Tortricoidea and Pyraloidea. They are able to put their eggs in the cryptobiont host. The hosts of chelonins are found in the grooves, galls, fruits, curled or rounded leaves and under bark. The physical and chemical effects of the substances produced by the host are important in its settlement and acceptance. The Parasitoid larvae encounter less problems in the host body and develop rapidly. The koinobionts allow the development of the host until the next cycle, that is, they allow the host larva to survive until the parasitoid larva becomes pupa. Cheloninae furn ish the most extreme examples of th is kind of delayed development (SHAw \& HudDLESTON 1991). Parasitoids are free-living as adults and parasitic as larvae; the larvae feed on their 
hosts, which are mostly insects of various developmental stages. Hosts do not survive and thus parasitoids play an important role as the regulatory agents for phytophagous insect population (LA SALLE \& GAULd 1993). While Ascogaster species are more common in bush areas and forest habitats, since Chelonus Panzer species are mostly tolerant of heat and drought, they are commonly found in steppe and grassland areas. Phanerotoma species are more abundant in arid regions or during dry seasons.

The Turkish fauna of Cheloninae is poorly known due to the low number of studies performed on the genus which were followed by studies all performed decades later: AydoĞdu (2008, 2016, 2017), AYdoĞdu \& BEYARSLAN (2002, 2006a, 2006b, 2007, 2009, 2011, 2012), Beyarslan (1985, 1995), BeyArslan et al. (2002), BEyARSLAN \& ŞAHIN (2018, 2019), Tobias (1986), Lozan (2005), ÖzGEN et al. (2012). On the other hand, important contributions to Turkish Chelonini fauna were made in the past in a number of studies. Some of these studies: 23 Cheloninae species were determined in the Eastern Anatolian region with 20 being new records for the region (AYDOĞDU 2016). More recently, 13 Chelonus species were identified from the Middle Anatolian region (AYDoĞdu 2017). AYDoĞdu \& BEYARSLAN (2006a) published 18 Microchelonus species from the Marmara, Western, and Middle Black Sea regions of Turkey and 8 of these species were reported for the first time from Turkey. 26 species of Ascogaster and Chelonus were recorded by the same researchers in the same region in 2007. AYDOĞDU \& BEYARSLAN (2009) determined 13 Phanerotomini species, and 6 of these were reported for the first time from Turkey. 13 Chelonus species were identified, and 5 of them are new records for the Turkish fauna (AYDoĞdu \& BEYARsLAn 2011). 18 Ascogaster species were recorded from Turkey and 2 of these are new to the Turkish fauna (AYDOĞDU \& Beyarslan 2012). 5 species of Chelonus, Phanerotoma, and Phanerotomella were recorded from the Turkish islands Imbros and Tenedos (BEYARSLAN et al. 2002). As a result of this study, 59 species belonging to 5 genera were identified. This research has been conducted in order to contribute to the Cheloninae fauna of Turkey. As a result, 16 species of Ascogaster, 18 species of Chelonus, 18 species of Microchelonus, 6 species of Phanerotoma and 1 species of Phanerotomella were recorded. 9 of these species are the first record for the fauna of Turkey.

\section{Material and methods}

Samples of adult Cheloninae were collected from different localities of Ardahan, Erzurum, Iğdır and Kars provinces of Turkey (Fig. 1). Sweeping nets, Malaise and light traps were used for material collection. Adults were separated from the other material with the help of an aspirator and were immediately killed in the absorption tubes with cigarette smoke. The collected material was brought to the laboratory in storage containers and in $70 \%$ ethylalcohol medium.

Preparation: Samples were in the laboratory, purified from ethylalcohol, dried and mounted on cards, in a way or card points that the important diagnistic characters are clearly visible. Samples are then labeled with locality labels.

Taxonomic examination: Comparison material in our collection and related literature (Huddleston 1984, Papp 1995, 2004, Shaw \& Huddleston 1991, Tobias 1976, 1986, 2008), van ACHTERBERG 1982, 1990) were utilized when evaluating the study materials. Information on parasitoids, hosts, general distributions (in terms of zoogeographical region) and parasitoids of species are given according Tobias (1986), Yu et al. (2016). In the text, the host plants of host species are shown in brackets. Examples are stored in 


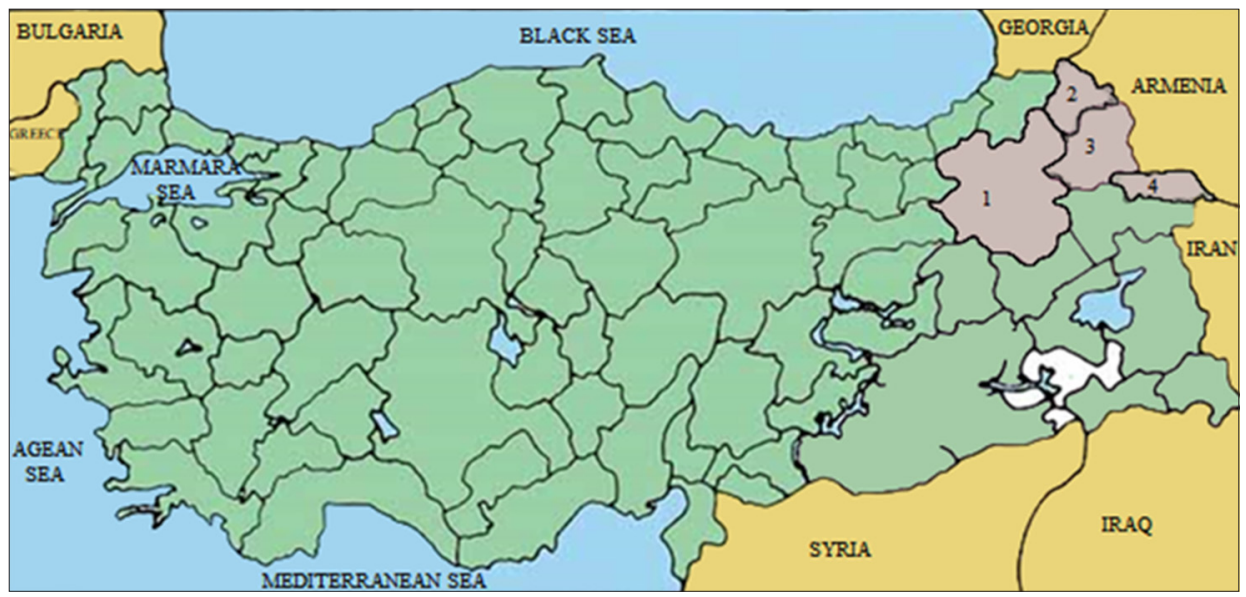

Fig. 1: Investigated provinces in the eastern Anatolian region of Turkey:

1. Erzurum, 2. Ardahan, 3. Kars and 4. Iğdır.

Trakya University, Faculty of Sciences, Department of Biology, Zoology collection. An asterisk $(*)$ was used within the text for the species that were recorded first in Turkey.

Taxonomical note: SzÉPLIGETI (1908) identified the genus Microchelonus. Until recently, it has been evaluated as a genus. KitTEL \& AUSTIN (2016) a molecular study on Chelonini revealed that Microchelonus is not a real genus and the real genus is Chelonus. However, in this study, we accept Microchelonus as a genus. Because we identified the species according to the old keys and system.

\section{Results}

Specimens of Cheloninae were collected from different habitats of Ardahan, Erzurum, Iğdır and Kars provinces of Turkey. In total, 32 species belonging 3 genera were determined. The distribution of the species by genera is as follows. Ascogaster Wesmael, 1835: 7, Chelonus Panzer, 1806: 10, Microchelonus Szépligeti, 1908: 15 species. The 32 species identified are new records for the fauna of research area and 9 of these species are the first record for the Turkish fauna.

\section{Subfamily: Cheloninae}

Genus: Ascogaster Wesmael, 1835

Ascogaster (Ascogaster) annularis (Nees, 1816)

Sigalphus annularis Nees, 1816

Ascogaster (Ascogaster) annularis: Curtis 1837

Material examined: Iğdır- Karakoyunlu, Taşburun, (3958'11"N, 44¹0'12"E), $851 \mathrm{~m}$ 26.6.2012, 1 万.

Host: Hymenoptera, Cynipidae: Dichomeris sp. [Cercis siliquastram], Diplolepis rosae (Linnaeus, 1758). Lepidoptera, Coleophoridae: Coleophora lutipennella (Zeller, 1838), Gelechiidae: Parachronistis albiceps (Zeller, 1839), Recurvaria leucatella 
(Clerck, 1759) [Malus domestica, Pyrus communis], R. nanella (Denis \& Schiffermüller, 1775) [Malus domestica], Stenolechia gemmella (Linnaeus, 1758), Oecophoridae: Batia lambdella (Donovan, 1793), Parametriotidae: Chrysoclista linneella (Clerck, 1759), Psychidae: Narycia duplicella (Goeze, 1783), Tortricidae: Pandemis cerasana (Hübner, 1786), P. heparana (Denis \& Schiffermüller, 1775) [Malus domestica], Spilonota ocellana (Denis \& Schiffermüller, 1775) [Malus domestica], Yponomeutidae: Yponomeuta malinella Zeller, 1838, Y. padella (Linnaeus, 1758) [Euonymus europaeus].

Distribution: Palaearctic.

Ascogaster (Ascogaster) bicarinata (Herrich-Schäffer, 1838)

Chelonus bicarinatus Herrich-Schäffer, 1838.

Ascogaster (Ascogaster) bicarinata: Marshall 1885

Synonym: Ascogaster caucasicus Kokoujev, 1895, Ascogaster mlokossewitschi Kokujev, 1895, Ascogaster rostratus Szépligeti, 1896), Ascogaster rufiventris Telenga, 1941.

Material examined: Erzurum- Köprüköy, Ilıcasu, (3958'01"N, 41ํ52'17"E), 1602 m, 20.6.2012, 1 ㅇ․

Host: Lepidoptera, Tortricidae: Archips rosana (Linnaeus, 1758).

Distribution: Palaearctic.

\section{Ascogaster (Ascogaster) bidentula Wesmael, 1835}

Material examined: Ardahan- Hanak, (41 $\left.15^{\prime} 40^{\prime \prime} \mathrm{N}, 42^{\circ} 51^{\prime} 10^{\prime \prime} \mathrm{E}\right), 2012 \mathrm{~m}, 23.6 .2012$,

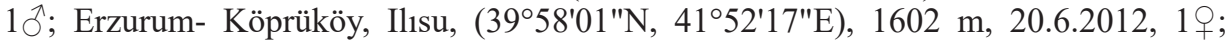
Kars- Sarıkamış, Erzurum yolu, 10km, (40¹5'03"N, 42³9'11"E), 2016 m, 23.6.2013, 1 오.

Host: Lepidoptera, Geometridae: Eupithecia venosata (Fabricius, 1787), Tortricidae: Archips sp. [Populus], A. rosana (Linnaeus, 1758), Epinotia cruciana (Linnaeus, 1761), Gypsonoma sociana (Haworth, 1811), Notocelia roborana (Denis \& Schiffermüller, 1775), Rhopobota myrtillana (Humphreys \& Westwood, 1845).

Distribution: Oriental, Palaearctic.

*Ascogaster (Ascogaster) canifrons Wesmael, 1835

Synonym: Ascogaster graniger Thomson, 1892, Ascogaster zernyanus Fahringer.

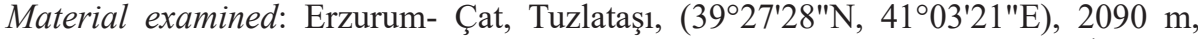

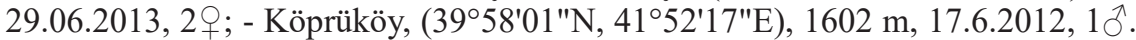

Host: Hymenoptera, Cynipidae: Andricus curvator Hartig, 1840. Lepidoptera, Geometridae: Eupithecia pyreneata Mabille, 1871, Tortricidae: Cydia pomonella (Linnaeus, 1758), C. splendana (Hübner, 1799), Endothenia quadrimaculana (Haworth, 1811), Eupoecilia angustana (Hübner, 1799), Gypsonoma dealbana (Frölich, 1828), Rhopobota naevana (Hübner, [1817]).

Distribution: Palaearctic.

*Ascogaster (Ascogaster) magnidentis Tobias, 1986

Material examined: Ardahan- Çı1dır, Eskibeyrehatun, (41 $\left.{ }^{\circ} 7^{\prime} 47^{\prime \prime} \mathrm{N}, 42^{\circ} 54^{\prime} 32^{\prime \prime E}\right), 1950$

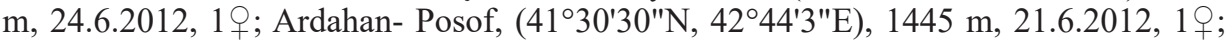

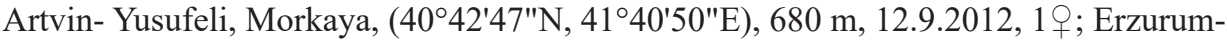

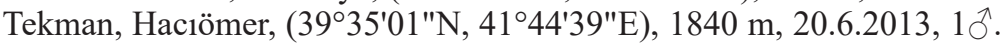


Host: Lepidoptera, Tortricidae: Cydia milleniana Razowski, 1991.

Distribution: Western Palaearctic.

* Ascogaster (Ascogaster) scabricula (Dahlbom, 1833)

Chelonus scabricula Zettel, 1987

Material examined: Ardahan- Çamlıçatak, (41 $\left.{ }^{\circ} 07^{\prime} 18^{\prime \prime} \mathrm{N}, 42^{\circ} 50^{\prime} 19^{\prime \prime} \mathrm{E}\right), 1850 \mathrm{~m}$,

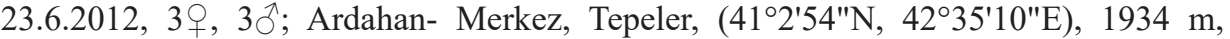
23.6.2012, 3 ㅇ, $1 \overbrace{}^{\top}$.

Host: Lepidoptera, Tortricidae: Cydia zebeana (Ratzeburg, 1840) [Larix europaea].

Distribution: Palaearctic.

Ascogaster (Ascogaster) varipes Wesmael, 1835

Synonym: Ascogaster jaroslawensis Kokujev, 1895

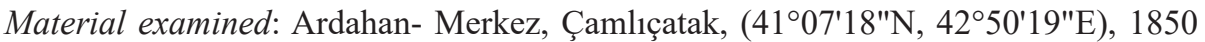
m, 23.6.2012, 1q; Kars- Kağızman, Aydın Kavak, (409'11"N, 43¹9'14"E), 1115 m, 20.6.2012, 1 ㅇ․

Host: Coleoptera, Curculionidae: Magdalis violacea (Linnaeus, 1758). Lepidoptera, Coleophoridae: Coleophora binderella (Kollar, 1832), Gelechiidae: Gelechia rhombella (Denis \& Schiffermüller, 1775), Tortricidae: Cydia compositella (Fabricius, 1775), C. grunertiana (Ratzeburg, 1868), C. pactolana (Zeller, 1840), C. zebeana (Ratzeburg, 1840), Endothenia gentianaeana (Hübner, 1799), Epinotia cruciana (Linnaeus, 1761).

Distribution: Palaearctic.

Genus: Chelonus Panzer, 1806

Subgenus: Chelonus s.str.

*Chelonus (Chelonus) ahngeri Tobias, 1966

Chelonus ahngeri Tobias, 1966.

Chelonus (Chelonus) ahngeri: Tobias 1972

Material examined: Kars- Kağıman, Morpet, $\left(40^{\circ} 15^{\prime} 16^{\prime \prime} \mathrm{N}, 42^{\circ} 58^{\prime} 36^{\prime \prime} \mathrm{E}\right), 1486$ m, 20.9.2012, $1 \overbrace{}^{\Uparrow}$.

Host: Unknown.

Distribution: Eastern Palaearctic.

Chelonus (Chelonus) annulatus (Nees, 1816)

Sigalphus annulatus Nees, 1816

Chelonus (Chelonus) annulatus: Tobias 1972

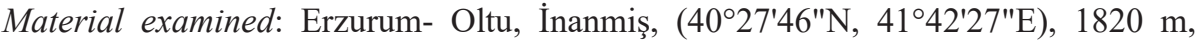
13.9.2012, 1 .

Host: Diptera, Tephritidae: Chaetostomella cylindrica (Robineau-Desvoidy, 1830) [Cirsium eriophorum], Trypeta acuticornis (Meigen 1824) [Cirsium eriophorum], Urophora eriolepidis (Loew, 1856) [Cirsium eriophorum]; Lepidoptera, Agonoxenidae: Chrysoclista linneella (Clerck, 1759). Parachronistis albiceps (Zeller, 1839). Recurvaria nanella ([Denis \& Schiffermüller], 1775), Stenolechia gemmella (Linnaeus, 1758), Oecophorinae: Batia lambdella (Donovan, 1793), Psychidae: Narycia duplicella (Goeze, 1783), Tortricidae: Dichrorampha petiverella (Linnaeus, 1758), Yponomeutidae: 
Yponomeuta malinellus Zeller, 1838, Y. padella (Linnaeus, 1758).

Distribution: Palaearctic

Chelonus (Chelonus) annulipes Wesmael, 1835

Chelonus annulipes Wesmael, 1835

Chelonus (Chelonus) annulipes: Abdinbekova 1971

Synonym: Chelonus falcatus Szépligeti, 1896, Sigalphus annulatus Nees von Esenbeck, C. G., 1816, Sigalphus sulcatus Szépligeti, 1901

Material examined: Erzurum- İspir, Petekli, (40²5'38"N, 4054'48"E), $1400 \mathrm{~m}$,

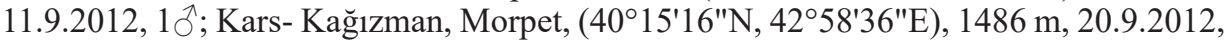
1 ㅇ, $1 \overbrace{}^{\top}$.

Host: Lepidoptera, Crambidae: Loxostege sticticalis (Linnaeus, 1761), Tortricidae: Cydia pomonella (Linnaeus, 1758), Pyralidae: Ostrinia nubilalis (Hübner, 1796) [Zea mays], Ephestia kuehniella Zeller, 1879, Noctuidae: Agrotis (Agrotis) segetum (DenisSchiffermüller, 1775), Eublemma pannonica (Freyer, 1840), Heliothis viriplaca (Hüfnagel, 1766), Spodoptera exigua (Hübner, 1808).

Distribution: Nearctic, Oriental, Eastern Palaearctic. Introduced into Canada-Ontario, Guam, Puerto Rico and Connecticut, Delaware, Florida, Illinois, Indiana, Iowa, Louisiana, Massachusetts, Michigan, New Jersey, New York, Ohio, Pennsylvania, Virginia and Wisconsin in USA for biological control of pests.

Chelonus (Chelonus) bidens Tobias, 1972

Material examined: Ardahan-Hanak, (41 $\left.{ }^{\circ} 15^{\prime} 40^{\prime \prime} \mathrm{N}, 42^{\circ} 51^{\prime} 10^{\prime \prime} \mathrm{E}\right), 2012 \mathrm{~m}, 23.6 .2012$,

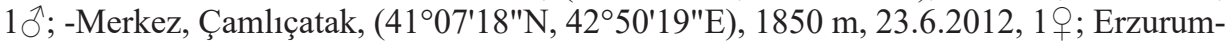

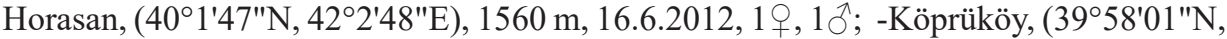

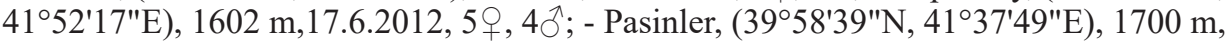

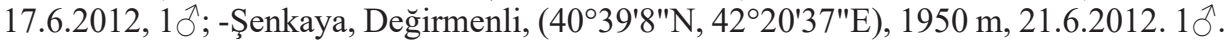

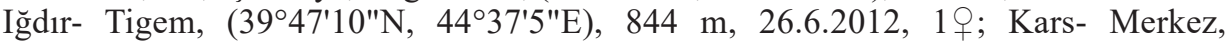

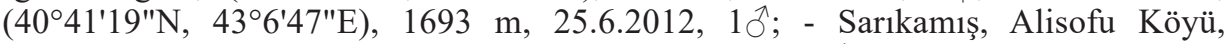

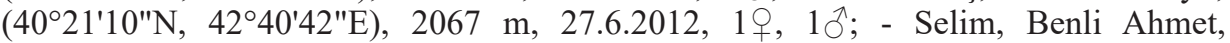
(402' $\left.40^{\prime \prime} \mathrm{N}, 42^{\circ} 54^{\prime} 39^{\prime \prime} \mathrm{E}\right), 1820 \mathrm{~m}, 27.6 .2012,1$.

Host: Unknown.

Distribution: Palaearctic.

Chelonus (Chelonus) cesa Koçak \& Kemal, 2013

Chelonus asiaticus Telenga, 1941 homonym of Chelonus asiatica Fahringer, 1932

Chelonus (Chelonus) asiaticus: Tobias 1972

Material examined: Ardahan, Merkez, Çamlıçatak, (41 $\left.{ }^{\circ} 07^{\prime} 18^{\prime \prime} \mathrm{N}, 42^{\circ} 50^{\prime} 19^{\prime \prime} \mathrm{E}\right), 1850$ m, 23.62012, 1 ㅇ.

Host: Unknown.

Distribution: Palaearctic.

Chelonus (Chelonus) corvulus Marshall, 1885

Chelonus corvulus Marshall, 1885

Chelonus (Chelonus) corvulus: Zhang \& Hong 2008.

Synonym: Chelonus suturatus Szépligeti, 1898. 


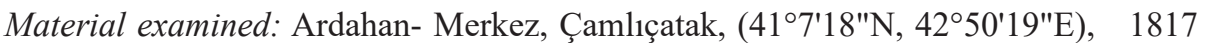

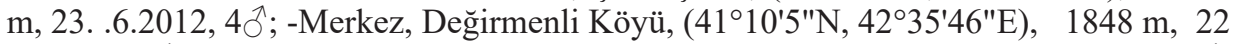

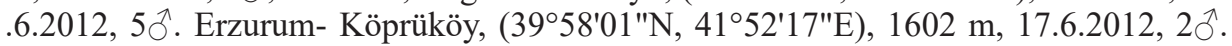
Kars- Sarıkmış, Alisofu Köyü, (40²1'10"N, 4240'42"E), 2067 m, 27.6.2012, $10^{\dagger}$.

Host: Lepidoptera. Coleophoridae: Coleophora caespititiella Zeller, 1839. Erebidae: Eublemma pannonica Freyer, 1840; Eublemma respersa Hübner, 1790. Gelechiidae: Scrobipalpa icella (F.v Röslerstam, 1841). Sesiidae: Chamaesphecia leucopsiformis (Esper, 1800). Noctuidae: Autographa gamma (Linnaeus, 1758); Shargacucullia verbasci (Linnaeus, 1758); ISpodoptera exigua (Hübner 1808). Tortricidae: Dichrorampha simpliciana (Haworth, 1811); Epiblema foenella (Linnaeus, 1758).

Distribution: Palaearctic.

Chelonus (Chelonus) dauricus Telenga, 1941

Chelonus dauricus Telenga, 1941

Chelonus (Chelonus) dauricus: Zhang 2008

Material examined: Ardahan -Çıldır, Y. Cambaz, 41 $\left.{ }^{\circ} 6^{\prime} 8^{\prime \prime} \mathrm{N}, 43^{\circ} 15^{\prime} 6^{\prime \prime} \mathrm{E}\right), 1980 \mathrm{~m}$,

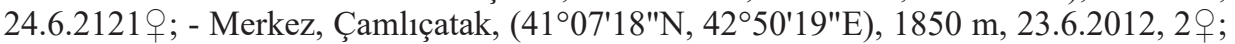

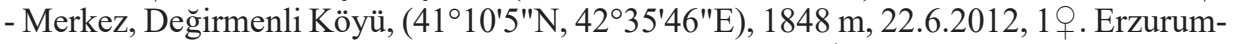

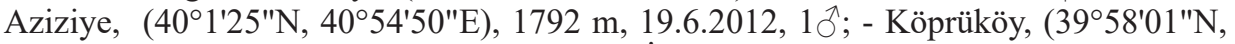

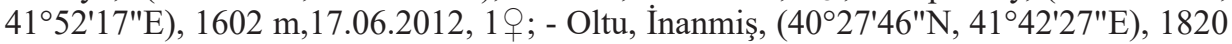

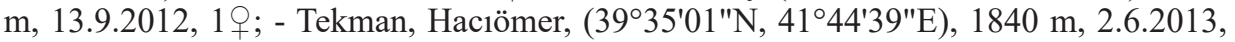
2ᄋ; Iğdır-Tigem, $\left(39^{\circ} 47^{\prime} 10^{\prime \prime} \mathrm{N}, 4^{\circ} 37^{\prime} 5^{\prime \prime} \mathrm{E}\right), 844 \mathrm{~m}, 26.6 .2012$, 1 \%; Kars- Merkez, $\left(40^{\circ} 41^{\prime} 19^{\prime \prime} \mathrm{N}, 43^{\circ} 6^{\prime} 47^{\prime \prime} \mathrm{E}\right), 1693 \mathrm{~m}, 25.6 .2012,1$, ; -Sarıkamış, Alisofu Köyü, (40²1'10"N, $\left.42^{\circ} 40^{\prime} 42^{\prime \prime E}\right), 2067 \mathrm{~m}, 27.6 .2012$, 4우 ; -Selim, Benliahmet, (40²9'40"N, 4254'39"E), $1820 \mathrm{~m}, 27.6 .2012,2$, $1 \jmath^{\lambda}$.

Host: Unknown.

Distribution: Palaearctic.

*Chelonus (Chelonus) jacobsoni Tobias, 1986

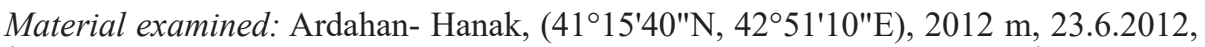

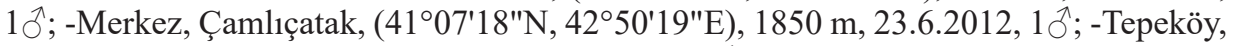

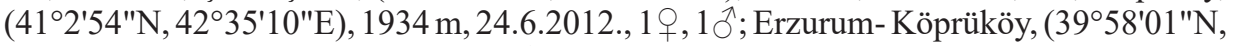
41 $\left.{ }^{\circ} 52^{\prime} 17^{\prime \prime E}\right), 1602 \mathrm{~m}, 17.06 .2012$, 1 \%; -Şenkaya, Sindıran, (40³7'12"N, 42²2'22"E),

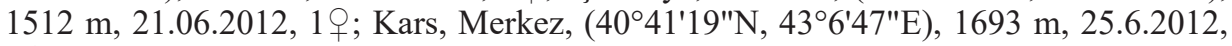
2 .

Distribution: Western Palaearctic.

*Chelonus (Chelonus) planiventris Tobias, 1960

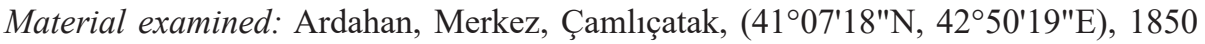
$\mathrm{m}, 23.6 .2012,2$.

Host: Unknown.

Distribution: Palaearctic. 
Chelonus (Chelonus) oculator (Fabricius, 1775)

Ichneumon oculator Fabricius, 1775

Chelonus (Chelonus) oculator: Abdinbekova 1971

Synonym: Anomala integra, Ichneumon scabrator Fabricius, 1793, Sigalphus cylindrus Nees von Esenbeck, 1816, Sigalphus mutabilis Nees von Esenbeck, C. G., 1816.

Material examined: Ardahan- Değirmenli Köyü, (41 $\left.{ }^{\circ} 10^{\prime} 5^{\prime \prime} \mathrm{N}, 42^{\circ} 35^{\prime} 46^{\prime \prime} \mathrm{E}\right), 1848$ m,

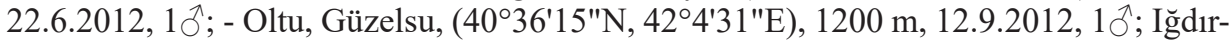

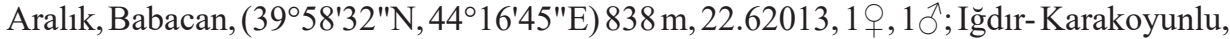

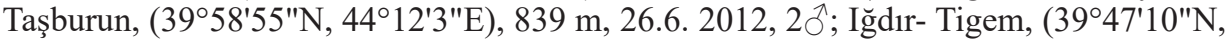

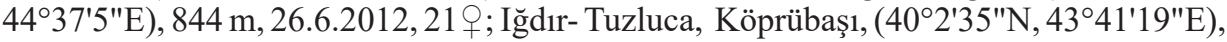
$1100 \mathrm{~m}, 20.9 .2012,1$.

Host: Lepidoptera, Coleophoridae: Coleophora anatipennella (Hübner 1796), Crambidae: Loxostege sticticalis (Linnaeus, 1761), Pyralidae: Ostrinia nubilalis (Hübner 1796), Ephestia kuehniella Zeller, 1879, Etiella zinckenella (Treitschke, 1832), Homoeosoma nebulella Denis-Schiffermüller, 1775, Tortricidae: Apotomis turbidana Hübner, [1825], Archips rosana (Linnaeus, 1758), Zeiraphera isertana (Fabricius, 1794), Noctuidae: Agrotis segetum Denis-Schiffermüller, 1775, Chortodes elymi (Treitschke, 1825), Helicoverpa armigera (Hübner, 1805), Heliothis peltigera (DenisSchiffermüller, 1775), H. viriplaca (Hüfnagel, 1766), Leucania loreyi (Duponchel, 1827) [Zea mays], Spodoptera exigua (Hübner 1808).

Distribution: Palaearctic Region.

Genus: Microchelonus Szépligeti, 1908

Subgenus: Microchelonus s.str.

Microchelonus (Microchelonus) atripes (Thomson, 1874)

Chelonus atripes Thomson, 1874

Microchelonus (Microchelonus) atripes: Papp 2014

Material examined: Kars-Sarıkamış, Asboğa, (40²4'03"N, 4243'35"E), 1900 m,

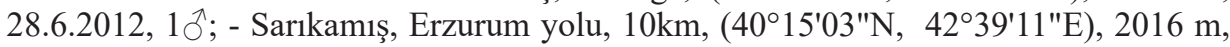
23.6.2013, 1 ㅇ․

Host: Lepidoptera, Coleophoridae: Coleophora alticolella Zeller, 1849; C. glaucicolella Wood, 1892.

Distribution: Palaearctic.

Microchelonus (Microchelonus) artus Tobias, 1986

Microchelonus artus Tobias, 1986

Microchelonus (Microchelonus) artus: Papp, 2014

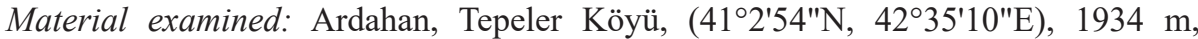
22.6.2012, 1 \% ; Kars- Arpaçay, Yalınçayır, (4048'11"N, 43ํ18'56"E), 1695 m, 24.6.2012,

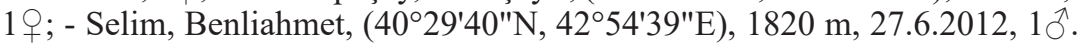

Host: Unknown.

Distribution: Western Palaearctic.

*Microchelonus (Microchelonus) kiritshenkoi (Tobias, 1976)

Chelonus (Microchelonus) kiritshenkoi Tobias, 1976

Microchelonus (Microchelonus) kiritshenkoi: Papp 2014 
Taxonomic note: Tobias described the new species as Microchelonus (Microchelonus) kiritshenkoi but he mentioned it as Chelonus (Microchelonus) kiritshenkoi in diagnostic key.

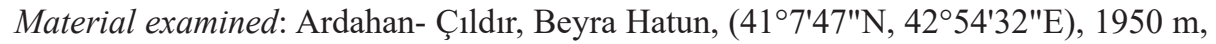

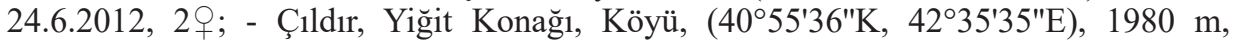

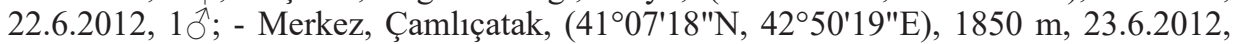

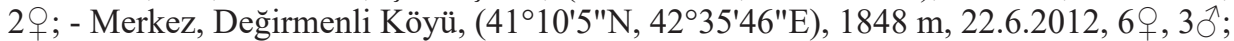

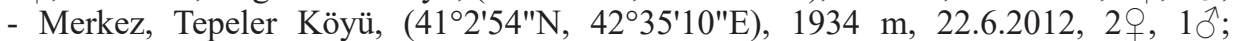

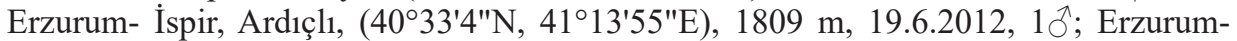
Narman, Yanıktaş, $\left(40^{\circ} 17^{\prime} 31^{\prime \prime} \mathrm{N}, 41^{\circ} 52^{\prime} 26^{\prime \prime} \mathrm{E}\right), 1602 \mathrm{~m}, 20.6 .2012$, 2ᄋ; Erzurum-

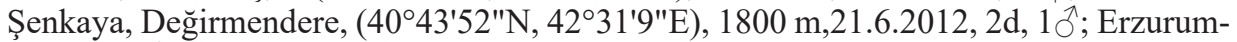
Şenkaya, İkizpınar, $\left(40^{\circ} 35^{\prime} 31^{\prime \prime} \mathrm{N}, 42^{\circ} 20^{\prime} 30^{\prime \prime E}\right), 1818 \mathrm{~m}, 21.6 .2012$, 19; Erzurum-

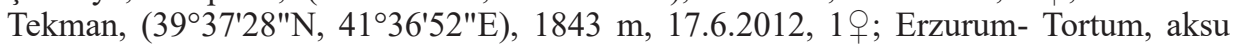
köyü, (40² $\left.5^{\prime} 41^{\prime \prime} \mathrm{N}, 41^{\circ} 36^{\prime} 12^{\prime \prime} \mathrm{E}\right), 2302 \mathrm{~m}, 16.6 .2012$, 1 ; ; Erzurum, Tortum, Yanıktaş, $\left(40^{\circ} 17^{\prime} 31^{\prime \prime} \mathrm{N}, 41^{\circ} 52^{\prime} 26^{\prime \prime E}\right), 1602 \mathrm{~m}, 20.6 .2012$, 1 웅 Iğdır- Tuzluca, Gaziler, (4006'36"N,

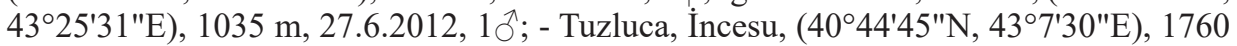

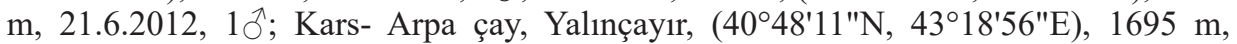
24.6.2012, 1우 ; Kağıman, Morpet, (40¹5'16"N, 4258'36"E), 1486 m, 20.6.2012, 19 .

Host: Unknown.

Distribution: Western Palaearctic.

Microchelonus (Microchelonus) kopetdagicus (Tobias, 1966)

Neochelonella kopetdagicus Tobias, 1966,

Microchelonus (Microchelonus) kopetdagicus: Papp 2014.

Material examined: Erzurum, Çat, Tuzlataş1, Erzurum-Çat-Tuzlataşı, (39²7'28"N, $\left.41^{\circ} 03^{\prime} 21^{\prime \prime E}\right), 2090 \mathrm{~m}, 29.06 .2013,29.06 .2013,2$ ㅇ․

Host: Unknown.

Distribution: Palaearctic.

Microchelonus (Microchelonus) longiventris (Tobias, 1964)

Neochelonella longiventris Tobias, 1964

Microchelonus (Microchelonus) longiventris: Papp 2014

Material examined: Ardahan- Çıldır, Eski Beyra Hatun, (41 $\left.{ }^{\circ} 7^{\prime} 47^{\prime \prime} \mathrm{N}, 42^{\circ} 54^{\prime} 32^{\prime \prime} \mathrm{E}\right)$,

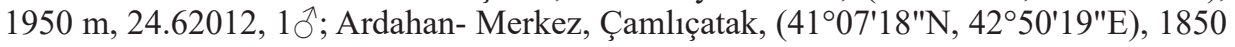
m, 23.6.2012, 1; Erzurum- Oltu, Yolboyu Köyü, (40³9'3"N, 429'4"E), 1114 m,

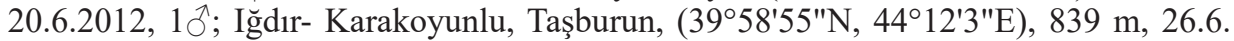

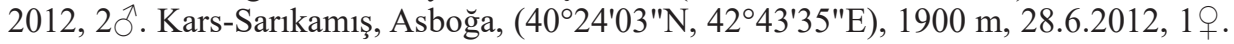

Host: Unknown.

Distribution: Palaearctic.

Microchelonus (Microchelonus) luzhetzkji (Tobias, 1966)

Material examined: Iğdır, Tigem, (3947'10"N, 44³7'5"E), 844 m, 26.6.2012, 1 ㅇ. Host: Hymenoptera, Yponomeutidae: Yponomeuta malinella Zeller, 1838; Y. padella (Linnaeus, 1758).

Distribution: Palaearctic. 
Microchelonus (Microchelonus) minifossa Tobias, 1986

Material examined: Ardahan, Merkez, Değirmenli Köyü, $\left(41^{\circ} 10^{\prime} 5^{\prime \prime} \mathrm{N}, 42^{\circ} 35^{\prime} 46^{\prime \prime} \mathrm{E}\right)$, $1848 \mathrm{~m}, 22.6 .2012,1 \delta^{\lambda}$.

Host: Unknown.

Distribution: Western Palaearctic.

Microchelonus (Microchelonus) nigritibialis (Abdinbekova, 1971)

Chelonus (Neochelonella) nigritibialis Abdinbekova, 1971

Microchelonus (Microchelonus) nigritibialis: Papp 2014.

Material examined: Iğdır- Tuzluca, Gaziler, $\left(40^{\circ} 06^{\prime} 36^{\prime \prime} \mathrm{N}, 43^{\circ} 25^{\prime} 31^{\prime \prime E}\right), 1035 \mathrm{~m}$, 27.6.2012, $1 \lesssim$.

Host: Unknown.

Distribution: Western Palaearctic.

*Microchelonus (Microchelonus) pectoralis (Tobias, 1976)

Material examined: Ardahan- Tepeler Köyü, (41 $\left.{ }^{\circ} 54^{\prime \prime N}, 42^{\circ} 35^{\prime} 10^{\prime \prime E}\right), 1934$ m,

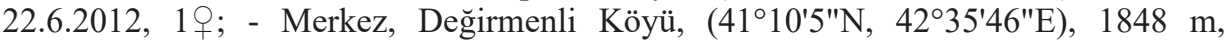
22.6.2012, 1; - Göle, Fakidemirkap1, (4051'9"N, 4247'31"E), 2050 m, 21.6.2012,

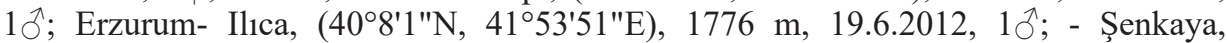
Değirmendere, $\left(40^{\circ} 43^{\prime} 52^{\prime \prime} \mathrm{N}, 42^{\circ} 31^{\prime} 9^{\prime \prime} \mathrm{E}\right), 1800 \mathrm{~m}, 21.6 .2012,2 \mathrm{~d}, 1{ }^{\top}$.

Host: Unknown.

Distribution: Palaearctic.

Microchelonus (Microchelonus) rostratus (Tobias, 1966)

Neochelonella rostratus Tobias, 1966

Microchelonus (Microchelonus) rostratus: Papp 2014

Material examined: Erzurum- İspir, Devedağı Köyü, (40³5'51"N, 41ํ18'38"E), 2231 $\mathrm{m}, 26.06 .2013,1{ }^{\Uparrow}$.

Host: Unknown.

Distribution: Palaearctic.

Microchelonus (Microchelonus) rugicollis (Thomson, 1874)

Chelonus rugicollis Thomson, 1874

Microchelonus (Microchelonus) rugicollis: Papp 2014

Material examined: Erzurum- Oltu, Yolboyu Köyü, (40³9'3"N, 429'4"E), 1114 m, 20.6.2012, $1 \overbrace{}^{\pi}$.

Host: Lepidoptera, Alucitidae: Alucita hexadactyla Linnaeus, 1758.

Distribution: Afrotropical, Palaearctic.

Microchelonus (Microchelonus) sulcatus (Jurine, 1807)

Material examined: Ardahan-Hanak, (41 $\left.{ }^{\circ} 15^{\prime} 40^{\prime \prime} \mathrm{N}, 42^{\circ} 51^{\prime} 10^{\prime \prime} \mathrm{E}\right), 2012 \mathrm{~m}, 25.6 .2012$,

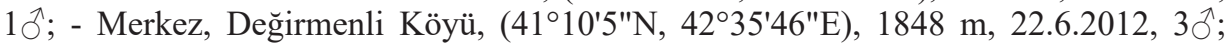

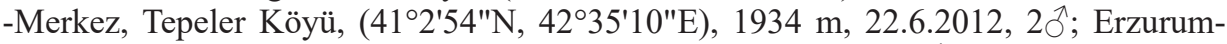

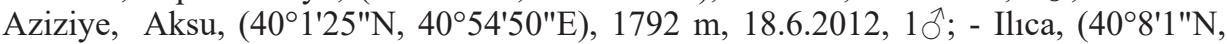




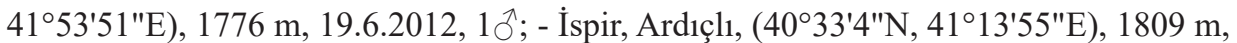

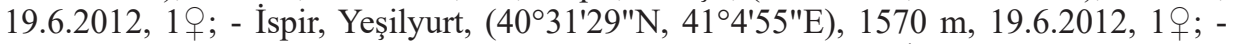

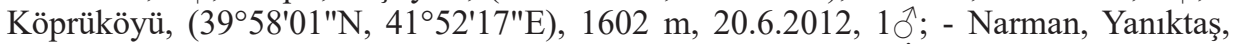
(40¹7'31"N, 4152'26"E), 1602 m, 20.6.2012, 20; - Şenkaya, İkizpınar, (40³5'31"N,

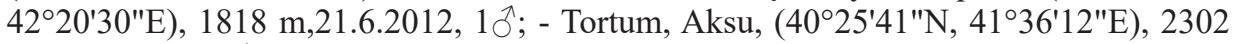

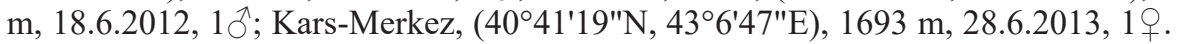

Host: Lepidoptera, Carposinadae: Carposina niponensis Walsingham, 1900, Coleophoridae: Coleophora tadzhikiella Danilevsky, 1955, Augasma aeratella (Zeller, 1839), Crambidae: Eudonia truncicolella (Stainton, 1849), Erebidae: Metachrostis dardouini (Boisduval, 1840), Gelechiidae: Pexicopia malvella (Hübner, [1805]), Pectinophora gossypiella (Saunders, 1844) [Gossypium sp.], Scrobipalpa ocellatella (Boyd, 1859), Mycetophilidae: Neoempheria striata (Meigen, 1818), Tortricidae: Rhyacionia buoliana (Denis \& Schiffmüller, 1775), Yponomeutidae: Prays citri (Milliére, 1873), Euhyponomeutoides albithoracellus Gaj, 1954 [Ribes nigrum].

Distribution: Palaearctic.

*Microchelonus (Microchelonus) vescus (Kokujev, 1899)

Chelonus vescus (Kokujev, 1899

Synonym: Chelonus minutus Szépligeti, 1898

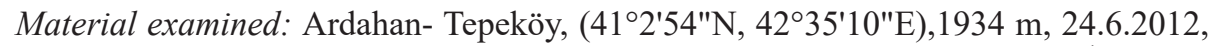

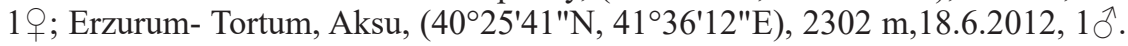

Host: Unknown.

Distribution: Palaearctic.

Subgenus: Parachelonus Tobias, 1995

\section{Chelonus (Parachelonus) pellucens (Nees, 1816)}

Sigalphus pellucens Nees, 1816

Microchelonus (Parachelonus) pellucens: Tobias 195.

Chelonus (Parachelonus) pellucens: Nees, 1834

Synonym: Chelonus alboannulatus Szépligeti, 1896, Chelonus nitens Reinhard, 1867, Chelonus pulchricornis Szépligeti, 1896, Chelonus varimaculatus Tobias, 1986.

Material examined: Ardahan- Hanak, (41 $\left.{ }^{\circ} 15^{\prime} 40^{\prime \prime} \mathrm{N}, 42^{\circ} 51^{\prime} 10^{\prime \prime} \mathrm{E}\right), 2012 \mathrm{~m}, 23.6 .2012$,

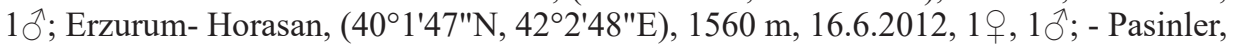

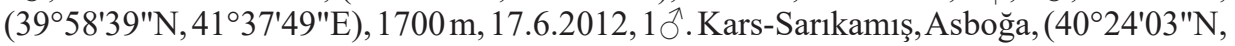
4243'35"E), $1900 \mathrm{~m}, 28.6 .2012,1$ ㅇ.

Host: Lepidoptera, Sesiidae: Bembecia hungarica (Tomala, 1910), B. ichneumoniformis ([Denis-Schiffermüller]1775), B. scopigera (ScopolI, 1763), Chamaesphecia euceraeformis (Ochsenheımer, 1816), C. tenthrediniformis (Denis-Schiffermüller, 1775).

Distribution: Palaearctic.

*Microchelonus (Parachelonus) starki (Telenga, 1953)

Chelonus starki Telenga, 1953

Chelonus (Parachelonus) starki: Tobias, 1995.

Material examined: Erzurum- İspir, Kırıkköyü, (40²0'13"N, 4057'3"E), 2030 m,

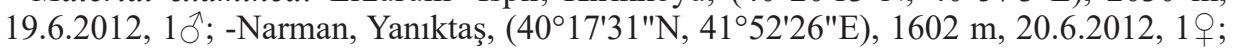




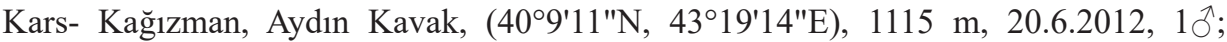
-Sarıkamış, Alisofu Köyü, (40²1'10"N, 4240'42"E), 2067 m, 27.6.2012, 1ㅇ.

Host: Lepidoptera, Sesiidae: Chamaesphecia bibioniformis (Esper, 1800), Paranthrene tabaniformis (Rottemburg, 1775), Synanthedon formicaeformis (Esper, 1782), S. myopaeformis (Borkhausen, 1789), S. tipuliformis (Clerck, 1759), Tortricidae: Cydia pomonella Linnaeus, 1758.

Distribution: Palaearctic.

\section{Discussion}

A total of 32 species were identified under the 3 genus of Cheloninae subfamily in the studied region. The distribution of the species by genera is as follows. Ascogaster: 7, Chelonus: 10 and Microchelonus: 15 (Fig. 2). 9 of these species (Ascogaster (Ascogaster) canifrons, A. (A.) magnidentis, A. (A.) scabricula, Chelonus (Chelonus) ahngeri, C. (C.) jacobsoni, C. (C.) planiventris, Microchelonus (Microchelonus) kiritshenkoi, M. (M.) vescus and $M$. (P.) starki) are the first record for the Turkish fauna. Up to now, 59 Cheloninae species have been recorded in Turkey. With this study, the number of Cheloninae species in Turkey was increased to 68.

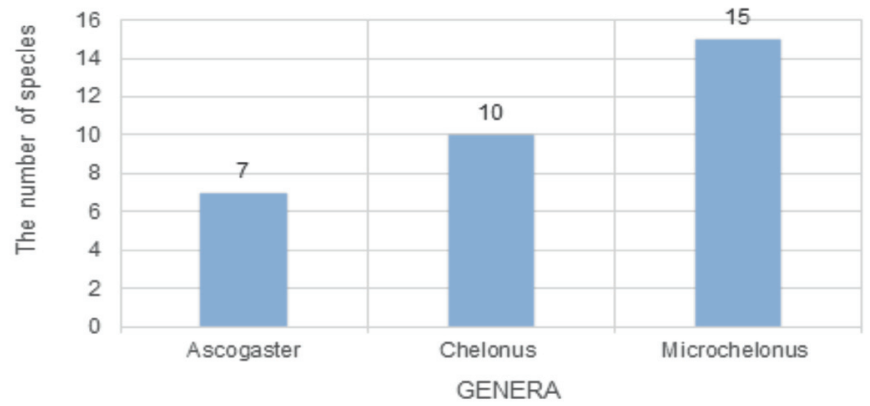

Fig. 2. The recorded genera and their number of species

As seen in Fig. 2, the genus represented by the most species is Microchelonus.

The species densities of the provinces surveyed are as follows Ardahan 20, Erzurum 21, Iğdır 7 and Kars 13 species. Erzurum is the province with the highest species density (Fig 3).

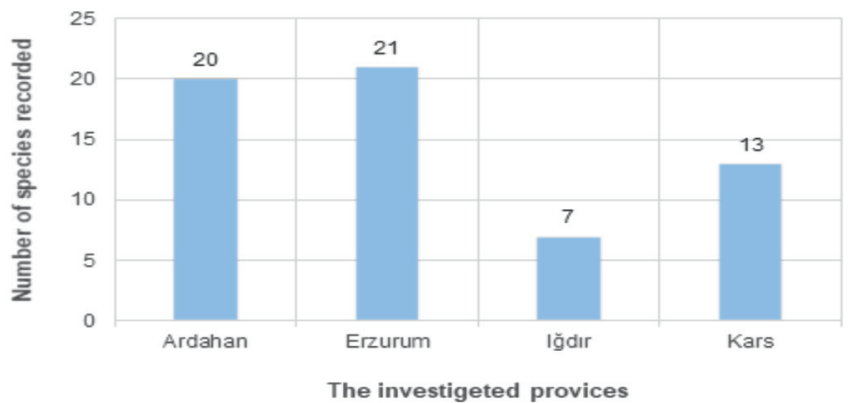

Fig. 3. The species densities of the provinces surveyed 
The most common species of research areas is Microchelonus (Microchelonus) kiritshenkoi. It was recorded from 16 localities.

C. (C.) annulipes is parasitoid of the following important pest species of Lepidoptera: Loxostege sticticalis (Crambidae), Ostrinia nubilalis [Zea mays], Agrotis (Agrotis) segetum, Cydia pomonella, Eublemma pannonica, Heliothis viriplaca, Spodoptera exigua (Noctuidae), Ephestia kuehniella (Pyralidae). Therefore, these species have been intruduced into the following countries for biological control of plant pest species: Ontario, Guam and Puerto Rico, Canada, Connecticut, Delaware, Florida, Illinois, Indiana, Iowa, Louisiana, Massachusetts, Michigan, New Jersey, New York, Ohio, Pennsylvania, Virginia, Wisconsin.

The above-mentioned hosts of $C$. (C.) annulipes are important pests of Turkish agriculture. This species be used for the same biological control of pests in Turkey (YU et al. 2016).

\section{Acknowledgements}

This research was supported by the Scientific and Technical Research Council of Turkey (TUBITAK) (Project-111T416). We thank these institutions for their financial support.

In addition, thanks to Dr. Fahrettin Özbey for preparing graphics.

\section{References}

AydoĞdu, M. 2008: A new species of the genus Chelonus Panzer, 1806 (Hymenoptera: Braconidae: Cheloninae) from Western Anatolia (Turkey). - Biologia 63 (2): 245-248.

AydoĞdu, M. 2016: Doğu Anadolu Bölgesi Cheloninae Förster. 1862 (Hymenoptera, Braconidae) faunas1 üzerine taksonomik bir araştırma Türkiye Biyolojik Mücadele Dergisi. - Turkish Journal of Biological Control 7(2): 123-138.

AydoĞdu, M. 2017: İç Anadolu Bölgesi Chelonus Panzer, 1806 (Hymenoptera, Braconidae, Cheloninae) Faunası Üzerine Taksonomik bir Araştırma. - Journal of Tekirdag Agricultural Faculty 14(02): 64-70.

AydoĞdu, M. \& Beyarslan A. 2002: The Chelonus Jurine (Hymenoptera: Braconidae: Cheloninae) species of the Marmara Region. - Turkish Journal of Zoology 26: 1-13.

AydoĞdu, M. \& Beyarslan, A. 2006a: Ascogaster disparilis Tobias, 1986 (Hymenoptera: Braconidae: Cheloninae), the second record for Palaearktik Region from Turkey. - Linzer biologische Beitrage 38(1): 393-396.

AydoĞdu, M. \& Beyarslan, A. 2006b: Microchelonus Szépligeti, 1908 (Hymenoptera: Braconidae: Cheloninae) species fron Marmara, Western and Blacksea regions of Turkey. -Linzer biologische Beitrage 38(1): 397-407.

AydoĞdu, M. \& Beyarslan, A. 2007: Parasitoid species of the genera Ascogaster and Chelonus (Hymenoptera: Braconidae: Cheloninae) from the Marmara, Western and Middle Black sea regions of Turkey. - Acta Entomologica Slovanica 15: 75-90.

AydoĞdu, M. \& Beyarslan, A. 2009: A rewiev the Phanerotomini (Hymenoptera: Braconidae: Cheloninae ) in Turkey, with a new host record for Phanerotoma (Bracotritoma) permixtella. - Biologia 64(4): 748756.

AydoĞdu, M. \& Beyarslan, A. 2011: Additional Notes on Chelonus Panzer, 1806 Fauna of Turkey with New Records (Hymenoptera, Braconidae, Cheloninae). - Journal of the Entomological Research Society 3: $75-81$. 
AydoĞdu, M. \& Beyarslan, A. 2012: A review of the genus Ascogaster Wesmael, 1835 (Hymenoptera, Braconidae, Cheloninae) in Turkey, with a new host record for Ascogaster Bicarinata (Herrich-Schäffer, 1838). - North-Western Journal of Zoology 8(1): 31-40.

Belokobylski, A. S. 1993: New taxonomic data on the braconid fauna (Hymenoptera, Braconidae) of Vietnam. - Russian Entomological Journal 2(2): 37-67.

Beyarslan, A. 1985: Die im Mittelmeergebiet der Türkei vertretenen Cheloninae-Arten (Hym: Braconidae) und ihre Verbreitung. - Turkish Journal of Zoology 9: 12-19.

Beyarslan, A. 1995: Für die Fauna der Türkei neu festgestellte Arten der Gattung Microchelonus Szépligeti, 1908 (Hymenoptera, Braconidae, Cheloninae). - Entomofauna 16(6): 121-136.

Beyarslan A., InAnÇ, F., Çetin, Ö. \& AydoĞdu, M. 2002: Braconiden von den Türkischen Inseln Imbros und Tenedos (Hymenoptera, Braconidae: Agaihidinae, Braconinae, Cheloninae, Microgastrinae). Entomofauna 23(15): 173-188.

Beyarslan A. \& ŞAHAN, Y. B. 2019: New species of Chelonini (Hymenoptera: Braconidae) from pistachio twig borer moth (Kermania pistaciella Amsel (Lep.: Tineidae)) in Gaziantep. - Turkish Journal of Zoology 43: 388-392. doi:10.3906/zoo-1805-21

Beyarslan, A. \& ŞAhin, F. 2018: Taxonomic Investigations on Braconidae (Hymenoptera) Fauna of Bitlis Nemrut Caldera and its around. - Munis Entomology \& Zoology 13(1): 292-308.

Huddleston, T. 1984: Palaearctic species of Ascogaster Wesmael (Hymenoptera: Braconidae). - Bulletin of the British Museum (Natural History). Entomology 49(5): 341-392.

Kaeslin, M., Wehrle, I., Gossliknaus-Birrgin, C., Wyler, T., Guggisberg, D., Sehittny, J. C. \& Lanzrein, B. 2005: Stage-dependent strategies of hosts invasion in the egg-larval parasitoid Chelonus inanitus. Journal of Insect Physiology 51: 287-296.

Kittel, R. N. \& Austin, A.D. 2016: Synopsis of Australian chelonine wasps (Hymenoptera: Braconidae: Cheloninae) with description of two new genera. - Austral Entomology 53: 183-202.

KoHL, F. F. 1905: Hymenopteren In Penther A. und E. Zederbauer, Ergebnisse einer naturwissenschaftlichen Reise zum Erdschias Dagh. (Klein Asien) - Annalen Naturhistorischen Hofmuseums Wien, 20: 227.

La Salle, J. \& Gauld, T. D. 1993: Hymenoptera and Biodiversity. - Centre for Agriculture and Biosciences International, England pp. 384.

Lozan, I. 2005: Cheloninae wasps (Hymenoptera: Braconidae, Cheloninae) from uplands of Turkey. Entomologist's Monthly Magazine, 141: 151-160.

Özgen, I., Bolu, H. \& Beyarslan, A. 2012: Chelonus flavıpalpıs Szépligei, 1896 and Miraxrufilabris haliday, 1833 (Hymenoptera: Braconidae): Two new larva-pupa parasitoids of pistachio twig borer Kermania pistaciella Amsel, 1964 (Lepidoptera: Ooinophilidae) with the parasitization ratios from Turkey. - Munis Entomology \& Zoology 7(1): 238-241.

PAPP, J. 1995: Revision of Wesmael's Chelonus species (Hymenoptera Braconidae Cheloninae). - Bulletın de l'Institut Royal des Sciences Naturelles de Belgique, Entomologie 65: 115-134.

Shaw, M. R., \& Huddleston, T. 1991: Classification and biology of Braconid wasps (Hymenoptera: Braconidae). - Handbooks for the Identification of British Insects 7: 1-126.

SzÉPligeti, G. 1908: Braconidae und ichneumonidae. - W Michaelson \& R Hartmeyer (eds.), Die Fauna Südwest-Australiens, Vol. 1. pp. 317- 324. Gustav Fischer, Jena.

TobIas, V. I. 1976: Braconids of the Caucasus (Hymenoptera, Braconidae). Opredeliteli po Faune SSSR No. 110: 1-286. Nauka Press, Leningrad, SSSR.

Tobias V.I. 1986: Family Braconidae. In: Medvedev G.S. (ed). Key to the insects of the European part of the USSR. Volume III, Hymenoptera Part IV. - Academy of Sciences of the USSR, New Delhi: Amerind Publishing. $908 \mathrm{p}$.

ToBIAS, V. I. 2008: Palaearctic species of the Microchelonus retusus group (Hymenoptera, Braconidae, Cheloninae). - Entomological Review 88: 1171-1191.

van Achterberg, C. 1982: Revisionary notes on Chelonus Jurine and Anomala Von Block Hymenoptera: Braconidae: Cheloninae). - Entomologische Berichte 42: 185-190.

van Achterberg, C. 1990: Revision of the Western Palearctic Phanerotomini (Hym: Braconidae). Zoologische Verhandelingen (Leiden) 255: 1-106, Figs: 1-360.

Yu D. S., van Achterberg, C. \& Horstmann, K. 2012: World Ichneumonoidea 2011. Taxonomy, Biology, Morphology and Distribution. Interactive catalogue on DVD/CDROM, Vancouver. Available from: http:// www.taxapad.com (Accessed 22. April, 2021) 